\title{
ON HIERARCHIES AND SYSTEMS OF NOTATIONS
}

\section{HILARY PUTNAM}

Introduction. By a "system of notations" we understand a (1-1)mapping, $M$, from a set $L$ (a segment of Cantor's second number class) onto a family, $F$, of disjoint nonempty sets. The members of $F$ may be, for example, sets of expressions, e.g. such expressions as $\omega$, $\omega \times 2, \omega^{2}, \epsilon_{0}$, etc. Without loss of generality we may assume that the sets in $F$ are sets of natural numbers. We say "without loss of generality" because expressions of the kind mentioned can always be replaced by numbers, using the device of gödel-numbering.

In the most common systems of notations, ${ }^{1}$ the number " 1 " is used as a name for the ordinal 0 , and $2^{x}$ is used to name the successor of the ordinal named by $x$. Thus $2,2^{2}, 2^{2^{2}}, \cdots$ are names, respectively, of $1,2,3, \cdots$. If $M$ is a mapping of the kind described, we think of the image of the ordinal $\alpha$ as the set of "notations" for $\alpha$, and we call this set $N_{\alpha}$. Thus we may write:

$$
M: \alpha \leftrightarrow N_{\alpha} .
$$

The reader may wonder why the set $N_{\alpha}$ is allowed to contain more than one number (i.e., more than one name or notation for the ordinal $\alpha$ ). The reason is that the possibility of having different names for the same ordinal, e.g. $\omega$ and $2^{\omega}$, or $\epsilon_{0}$ and $\omega^{e}{ }_{0}$, arises in all of the common systems.

The most important restriction upon the mapping $M$ is that it should in some sense be inductively defined. This leads us to an abstract "system of notations," $D$, which we present below. First we need the

Definition. Let $\cdots P, x, \cdots$ be a formula of second-order arithmetic containing one free variable $P$ for a two-term relation between numbers, one free variable $x$ for numbers, arbitrary bound number variables, but no free or bound higher-order variables except $P$. (The constants are to designate numbers and recursive functions and predicates.) Then the mapping $P \leftrightarrow\{x \mid \cdots P, x, \cdots\}$ associates with every diadic relation $P$ between natural numbers a set of numbers, namely the set of all numbers $x$ satisfying $\cdots P, x, \cdots$. Such a mapping is here called an arithmetic operation. ${ }^{2}$

Received by the editors October 29, 1962.

${ }^{1}$ Cf. [C], [CK], [KR], [P].

${ }^{2}$ If $\cdots P, x \cdots$ be allowed to contain second-order bound variables, then the operation may be classified as $\Sigma_{1}^{1}, \Pi_{1}^{1}, \Sigma_{2}^{1}, \ldots$ etc., according to the number and quality of these. (Cf. footnote 3 below.) 
The following is the system $D$ :

(1) If $\alpha=0$, then $N_{\alpha}=\{1\}$.

(2) If $N_{\beta}$ has already been defined for all $\beta \leqq \alpha$, then $N_{\alpha+1}$ $=\left\{2^{x} \mid x \in N_{\alpha}\right\}$.

(3) If $\alpha$ is a limit number and $N_{\beta}$ has already been defined for all $\beta<\alpha$, then $N_{\alpha}=\phi\left[\hat{x} y\left(x \leqq{ }_{\alpha} y\right)\right]$, where $\phi$ is an arithmetic operation which leads from 2-term relations between natural numbers to sets of natural numbers.

In the definition of $D$, part (3), we have used $x \leqq{ }_{\alpha} y$ as an abbreviation for the 2-term relation (depending on $\alpha$ ):

$$
(\exists \beta)(\exists \gamma)\left(x \in N_{\beta} \& y \in N_{\gamma} \& \beta \leqq \gamma<\alpha\right) .
$$

We also impose the following:

Restrictions on the choice of $\phi$.

(1) The sets $N_{\alpha}$ defined above must be disjoint.

(2) There must be a limit number $\alpha$ such that $N_{\beta} \neq \varnothing$ for $\beta<\alpha$, and $N_{\beta}=\varnothing$ for $\alpha \leqq \beta$ (we think of $\alpha$ as "the least ordinal for which there is no notation in $D^{\prime \prime}$ ).

$D$ is, of course, not a single system but a schema for systems (determining a single system for each admissible choice of $\phi$ ). In fact, it is easily shown that all so far proposed systems are of the form $D$.

Let $C_{D}={ }_{\mathrm{df}} \cup N_{\alpha}$. We shall show that $C_{D} \in \Sigma_{2}^{1} \cap \Pi_{2}^{1}$. Our short proof of this fact replaces the lengthy and intricate argument used by Kreider and Rogers in [KR], since their main result is a special case of ours. If we place certain further restrictions on $\phi$, we can give the usual definition of the sets ${ }^{4} \mathfrak{S}_{n}$ for $n \in C_{D}$. We shall show that these $\mathfrak{S}_{n} \in \Sigma_{2}^{1} \cap \Pi_{2}^{1}$. Even if we use a "jump operation" in $\Sigma_{2}^{1} \cap \Pi_{2}^{1}$ instead of the "one-function-quantifier jump operation" (defined below), the $\mathfrak{W}_{n}$ are still in $\Sigma_{2}^{1} \cap \Pi_{2}^{1}$. Also the $\mathfrak{S}_{n}$ are still in $\Sigma_{2}^{1} \cap \Pi_{2}^{1}$ even if we let the operation $\phi$ be in $\Sigma_{2}^{1} \cap \Pi_{2}^{1}$, instead of restricting it to be arithmetic.

Thus we have a very strong closure property of the class $\Sigma_{2}^{1} \cap \Pi_{2}^{1}$ : we cannot get out of this class by iterating a jump operation through all the ordinals for which we have names in a system $D$ unless (i) we are using a jump operation which is not in $\Sigma_{2}^{1} \cap \Pi_{2}^{1}$ (i.e., which already goes out of $\Sigma_{2}^{1} \cap \Pi_{2}^{1}$ in one jump) or (ii) we have already used an operation $\phi$ which is not in $\Sigma_{2}^{1} \cap \Pi_{2}^{1}$ in defining $D$.

\footnotetext{
${ }^{3}$ A set definable in second order number theory is said to belong to the family $\Sigma_{n}$ if it can be defined with $n$ function quantifiers, the first of which is existential (in some prenex form), and is said to belong to the family $\Pi_{n}^{1}$ if it can be defined with $n$ function quantifiers the first of which is universal. ("function quantifier" $=$ second order quantifier.)
}

' Cf. $\left[\mathrm{K}_{1} ; \mathrm{P}\right]$. 
The set $\mathfrak{W}_{n}$ are the complete sets of the various "hyperdegrees." Thus, our result may be expressed by saying that even if the series of "hyperdegrees" is extended through $D$, we do not get any degrees for three-function-quantifier sets. Similarly, we shall show that we cannot get analogues of hyperdegrees for all the analytic sets by using an analytic jump operation and an analytic operation $\phi$.

Classification of $C_{D}$.

Theorem 1. Let $D$ be defined as above, and let $C_{D}=\cup N_{\alpha}$. Then $C_{D} \in \Sigma_{2}^{1} \cap \Pi_{2}^{1}$.

Proof. We have

$n \in C_{D} \Leftrightarrow(\exists$ a simple-ordering $f)(\exists$ a 2-place function $g)(h)\{[h$ is a nonincreasing $f$-chain $\Rightarrow(\exists x)(h(x)=h(x+1))] \&(y)$ (the least element of $f=y \Rightarrow(z)(g(y, z)=1 \Leftrightarrow z=1)) \&(y)(z)$ ( $z$ is the $f$-successor of $y \Rightarrow\{w \mid g(z, w)=1\}=\left\{2^{w} \mid g(y, w)\right.$ $=1\}) \&(y)(y$ is a limit element of $f \Rightarrow\{w \mid g(y, w)=1\}$ $=\phi[\hat{x} \hat{z}((\exists v)(\exists u)(g(u, x)=1 \& g(v, z)=1 \&(u f$-precedes $v \vee u=v) \& v f$-precedes $y))]) \&(\exists x)(g(x, n)=1)\}$.

Here we have used a well-ordering of all the integers in place of a segment of the second number class, and we have used the mapping

$$
x \leftrightarrow\{y \mid g(x, y)=1\}
$$

as our mapping $M$ (obviously without loss of generality). The prefix is of the form $\exists \exists \forall$ and the reader can readily verify that the matrix involves only ordinary number quantifiers. In fact (1) becomes:

$$
\begin{aligned}
n \in C_{D} \leftrightarrow & (\exists f)(\exists g)(h)[(x) f(x, x)=1 \&(y)(z)(f(y, z)=1 \& f(z, y)=1 \\
& \Rightarrow y=z) \&(x)(y)(f(x, y)=1 \bigvee f(y, x)=1) \&(x)(y)(z) \\
& (f(x, y)=1 \& f(y, z)=1 \Rightarrow f(x, z)=1) \&((x) f(h(x+1), \\
& h(x))=1 \Rightarrow(\exists x) h(x)=h(x+1)) \&(y)((z) f(y, z)=1 \\
& \Rightarrow(z)(g(y, z)=1 \Leftrightarrow z=1)) \&(y)(z)((f(y, z)=1 \& y \neq z \\
& \&(w)(f(y, w)=1 \& w \neq y \Rightarrow f(z, w)=1)) \Rightarrow(w)(g(z, w) \\
& \left.\left.=1 \Leftrightarrow(\exists x)\left(w=2^{x} \& g(y, x)=1\right)\right)\right) \&(y)((z) f(z, y)=1 \\
& \& z \neq y \Rightarrow(\exists w)(z \neq w \& f(z, w)=1 \& w \neq y \& f(w, y) \\
& =1)) \Rightarrow(w)(g(y, w)=1 \Leftrightarrow w \in \phi[\hat{x} \hat{z}((\exists u)(\exists v)(g(u, x) \\
& =1 \& g(v, z)=1 \& f(u, v)=1 \& f(v, y)=1 \& v \neq y))])) \\
& \&(\exists x) g(x, n)=1] .
\end{aligned}
$$


Contracting quantifiers, ${ }^{5}$ we have $C_{D} \in \Sigma_{2}^{1}$.

To prove that $C_{D} \in \Pi_{2}^{1}$ we argue similarly, using instead of (1):

$n \in C_{D} \Leftrightarrow(\boldsymbol{\forall}$ simple ordering $f)(g)\{[(h)(h$ is a nonincreasing $f$-chain $\Rightarrow(\exists x) h(x)=h(x+1) \& \cdots($ as in $(1)) \cdots \&(y)(y$ is a limit element of $f \Rightarrow\{w \mid g(y, w)=1\}=\phi[\cdots$ as in (1) ‥] $]$ \& $(\exists x)(y) g(x, y) \neq 1] \Rightarrow(\exists x) g(x, n)=1\}$.

Note that the clause " $(\exists x)(y) g(x, y) \neq 1$ " says that $f$ reaches the least ordinal for which there is no notation in $D$ !

Bringing out the quantifier $(h)$ (which comes out, of course, as an existential quantifier), and contracting quantifiers, we have $C_{D} \in \Pi_{2}^{1}$. Thus $C_{D} \in \Sigma_{2}^{1} \cap \Pi_{2}^{1}$. Q.E.D.

Theorem 2. If, in the definition of $D$, we allow $\phi$ to be a $\Sigma_{2}^{1} \cap \Pi_{2}^{1}$ operation, it remains the case that $C_{D} \in \Sigma_{2}^{1} \cap \Pi_{2}^{1}$.

Proof. In the proof that $C_{D} \in \Sigma_{2}^{1}$ we note that the quantifier (h) can be confined to the clause $((x) f(h(x+1), h(x))=1 \Rightarrow(\exists x) h(x)$ $=h(x+1))$. Then the function quantifiers in $\phi$ can be moved ahead of the number quantifiers by the devices of $\left[\mathbf{K}_{\mathbf{1}}\right]$ and finally brought out. Although $\phi$ occurs in a biconditional, the quantifiers can be brought out in the order $\exists \forall$ by splitting the biconditional into a pair of conditionals and using the $\exists \forall$ form of $\phi$ when $\phi$ occurs in the consequent and the $\forall \exists$ form when $\phi$ occurs in the antecedent. That is, $(w)(g(y, w)=1 \Leftrightarrow w \in \phi[\cdots])$ becomes

$$
(w)\left[\left(g(y, w)=1 \Rightarrow(\exists \alpha)(\beta)\left(\psi_{1}\right) \&\left((\delta)(\exists \lambda) \psi_{2} \Rightarrow g(y, w)=1\right)\right],\right.
$$

where $w \in \phi[\cdots] \Leftrightarrow(\exists \alpha)(\beta) \psi_{1} \Leftrightarrow(\delta)(\exists \lambda) \psi_{2}$. Hence the original expression becomes

$$
(w)(\exists \alpha)(\exists \delta)(\beta)(\lambda)\left[\left(g(y, w)=1 \Rightarrow \psi_{1}\right) \&\left(\psi_{2} \Rightarrow g(y, w)=1\right)\right] .
$$

Bringing out the function quantifiers and contracting we have $\boldsymbol{\exists} \boldsymbol{\forall}$ in front of the whole formula (except for the $(h)$ which we confined). Now we bring out $(h)$ and contract again, and we have $C_{D} \in \Sigma_{2}^{1}$. The argument is similar for $C_{D} \in \Pi_{2}^{1}$. Note that, since we have to exploit the fact that $\phi$ can be written in both ways, we would not get a better result by assuming that $\phi \in \Sigma_{1}^{1}$ or $\phi \in \Pi_{1}^{1}$ than we do by assuming that $\phi \in \Sigma_{2}^{1} \cap \Pi_{2}^{1}$. Generalizing this argument, we also see that if $\phi \in \Sigma_{n}^{1} \cap \Pi_{n}^{1}$, $n>2$, then $C_{D} \in \Sigma_{n}^{1} \cap \Pi_{n}^{1}$.

The sets $\mathfrak{S}_{n}$. Suppose a set $A$ is "one-function-quantifier" in a set $B$, i.e., say $x \in A \Leftrightarrow(f)(\exists y)(\cdots B \cdots)$ (that the number quanti-

r Cf. [ $\left.K_{1}\right]$, also [KR, p. 346]. 
fiers can be reduced to one when function quantifiers are present is shown in $\left.\left[\mathrm{K}_{1}\right]\right)$. Since $(\exists y)(\cdots B \cdots)$ says that $x$ belongs to a set which is recursively enumerable in $B, f$, we can write this as $x \in A$ $\Leftrightarrow(f)(\exists y) T^{\gamma, B}(g(x), g(x), y)$ for a suitable primitive recursive $g$. Thus the set $\left\{x \mid(f)(\exists y) T^{\prime, B}(x, x, y)\right\}$ is a complete one-function-quantifier form in $B$, i.e., every set which is one-function-quantifier in $B$ is recursive in this set (and if $A$ is $\Pi_{1}^{1}$ in $B, A$ is even many-one reducible to this set). This justifies calling the operation

$$
B \leftrightarrow\left\{x \mid(f)(\exists y) T^{f, B}(x, x, y)\right\}
$$

the complete one-function-quantifier jump operation (or, more simply, the hyper-jump operation). We shall use $D$ to iterate this operation transfinitely many times. First we impose the following.

\section{Further restrictions on $\phi$.}

(1) If $\alpha$ is a limit number, $N_{\alpha}$ must not contain any power of 2.

(2) There must exist recursive functions $g, k$ such that if $\alpha$ is a limit number and $x \in N_{\alpha}$, then $g(x) \in N_{\beta}$ for some limit number $\beta \leqq \alpha$, and $^{6}\{k(x)\}$ provides an order preserving cofinal mapping (see below) from $U_{\gamma<\beta} N_{\gamma}$ into $U_{\gamma<\alpha} N_{\gamma}$.

Let us write $|x|=\alpha$ for $x \in N_{\alpha}$. Then (2) means that

(i) if $y \in U_{\gamma<\beta} N_{\gamma}$ then $\{k(x)\}(y)$ is defined and $\{k(x)\}(y) \in N_{\alpha}$ (into property);

(ii) if $y, z \in U_{\gamma<\beta} N_{\gamma}$, then $|y| \leqq|z| \Leftrightarrow|\{k(x)\}(y)| \leqq|\{k(x)\}(z)|$ (order-preserving property);

(iii) for each $y \in U_{\gamma<\alpha} N_{\gamma}$, there is a $z \in U_{\gamma<\beta} N_{\gamma}$ such that $|y|$ $\leqq|\{k(x)\}(z)|$ (cofinal property).

Intuitively, the meaning of these restrictions is that it should be possible to effectively distinguish notations for successor ordinals from those for limit ordinals and to associate an order-preserving cofinal recursive sequence from $\mathrm{U}_{\gamma<\beta} N_{\gamma}$ into $\mathrm{U}_{\gamma<\alpha} N_{\gamma}$ (for some effectively specified $\beta$ ) with each (notation for $a$ ) limit ordinal $\alpha$. We allow $\alpha=\beta$ in restriction (2), because otherwise it would not be possible to have "recursively regular" ordinals."

We now define the set $\mathfrak{S}_{n}$ (obtained by iterating the hyper-jump operation through $D$ ) as follows:

(1) $\mathfrak{S}_{1}=\varnothing$.

(2) If $n \in D$, then $\mathfrak{\bigvee}_{2^{n}}=\left\{x \mid(f)(\exists y) T^{f} \mathfrak{\$}_{n}(x, x, y)\right\}$.

(3) If $n \in D, n$ is not a power of 2 , then

- $\{k(x)\}$ is the symbol for the $k(x)$ th partial recursive function in the standard enumeration.

${ }^{7}$ Cf. [KR; P]. [P] also defines the system $C$ in a way which makes it clear that this system is of the form $D$. 


$$
\mathfrak{W}_{n}=\left\{2^{x} 3^{y} \mid x \in \oiint_{\{k(n)\}(y)} \& y \in \bigcup_{\gamma<|0(n)|} N_{\gamma}\right\} .
$$

[Note that if $|n|$ is a limit ordinal, (3) insures that $\mathfrak{W}_{m}$ shall be recursive in $\mathfrak{W}_{n}$ for each $m$ in the appropriate "fundamental sequence" (provided by $\{k(n)\}(y)$ as $y$ runs through the notations for ordinals less than $|g(n)|)$.]

We see that each $\mathfrak{W}_{2}{ }^{n}$ comes from $\mathfrak{W}_{n}$ by the hyper-jump operation. And by an easy transfinite induction $\mathfrak{W}_{n}$ is recursive in $\mathfrak{W}_{m}$ for $|n|<|m|$.

Theorem 3. For each $n \in D$, the set $\mathfrak{S}_{n} \in \Sigma_{2}^{1} \cap \Pi_{2}^{1}$.

Proof. It suffices to write

$$
\begin{aligned}
x \in \mathfrak{W}_{n} \Leftrightarrow & (\exists f)(\exists g)\left(\exists g^{\prime}\right)(h)[\cdots \text { entire matrix of }(2) \cdots \\
& \&(w) g^{\prime}(1, w) \neq 1 \&(y)(z)\left(g\left(y, 2^{z}\right)=1 \Rightarrow(v)\left(g^{\prime}\left(2^{z}, v\right)=1\right.\right. \\
& \left.\left.\Leftrightarrow(\alpha)(\exists y) T^{\alpha, P}(v, v, y)\right)\right) \&(y)(z)(y \text { is a limit element of } \\
& f \& g(y, z)=1 \Rightarrow\left\{w \mid g^{\prime}(z, w)=1\right\}=\left\{J(w, v) \mid g^{\prime}(\{k(z)\}\right. \\
& (v), w)=1 \&(\exists a)(\exists b)(f(a, b) \& a \neq b \& g(b, g(z))=1 \\
& \left.\& g(a, v)=1\}) \& g^{\prime}(n, x)=1\right] .
\end{aligned}
$$

In (4) above " $P$ " abbreviates " $\left\{v \mid g^{\prime}(z, v)=1\right\}$. "

Here again we have used a well-ordering of all the integers instead of a segment of the second number class. We have used the mapping

$$
x \leftrightarrow\{y \mid g(x, y)=1\}
$$

as our mapping $\alpha \leftrightarrow N_{\alpha}$; and we have used the mapping

$$
y \leftrightarrow\left\{z \mid g^{\prime}(y, z)=1\right\}
$$

as our mapping $y \leftrightarrow \mathfrak{S}_{y}$. Writing out the $T^{\alpha, P}$ predicate in full and putting $g^{\prime}(z, w)=1$ and $g^{\prime}(z, w) \neq 1$ for $P(w)$ and $\bar{P}(w)$ whenever these occur in the full form of the predicate $T^{\alpha, P}(v, v, y)$, we see that the argument of Theorem 2 applies here. So, once again we confine $(h)$ to the single clause in which it occurs, split the biconditional involving $T^{\alpha, P}$ into a pair of conditionals, and then bring out the function quantifiers in the desired $\exists \forall$ order. Thus $\mathfrak{S}_{n} \in \Sigma_{2}^{1}$. The proof that $\mathfrak{\$}_{n} \in \Pi_{2}$ is exactly similar, writing

$$
\begin{aligned}
x \in \mathfrak{W}_{n} \Leftrightarrow & (f)(g)\left(g^{\prime}\right)\{[(h)(h \text { is a nonincreasing } f \text {-chain } \Rightarrow(\exists x) h(x) \\
& =h(x+1)) \& \cdots \text { as in }(4) \cdots \&(\exists x)(y) g(x, y) \neq 1] \\
& \left.\Rightarrow g^{\prime}(x, x)=1\right\} .
\end{aligned}
$$

Finally, we note that the argument again generalizes to show that if the operation 


$$
B \leftrightarrow(\alpha)(\exists y) T^{\alpha, B}(x, x, y)
$$

is replaced by an operation which leads (uniformly) from $B$ to a set which is in $\Sigma_{n}^{1} \cap \Pi_{n}^{1}$ form relative to $B, n \geqq 2$, then the sets $\mathfrak{W}_{n}$ will be in the class $\Sigma_{n}^{1} \cap \Pi_{n}^{1}$.

REMARK ADDED FEBRUARY 5, 1963. The method of this paper yields an immediate proof that if $B \in \Sigma_{2}^{1} \cap \Pi_{2}^{1}$ then the hyperjump of $B$ also $\in \Sigma_{2} \cap \Pi_{2}$. Namely, it suffices to define

$n \in$ hyperjump $(B)$

$$
\begin{gathered}
\leftrightarrow(\exists f)(\exists g)[(x)(f(x)=1 \vee f(x)=0) \&(x)(g(x)=1 \vee g(x)=0) \\
\&(x)(f(x)=1 \Leftrightarrow B(x)) \&(x)(g(x)=1 \\
\left.\left.\Leftrightarrow(h)(\exists y) T^{h, f}(x, x, y)\right) \& g(n)=1\right] \\
\Leftrightarrow(f)(g)[(x)(f(x)=1 \vee f(x)=0) \&(x)(g(x)=1 \vee g(x)=0) \\
\&(x)(f(x)=1 \Leftrightarrow B(x)) \&(x)(g(x)=1 \\
\left.\left.\Leftrightarrow(h)(\exists y) T^{h, f}(x, x, y)\right) \cdot \Rightarrow \cdot g(n)=1\right] .
\end{gathered}
$$

Splitting the biconditionals into pairs of conditionals and proceeding with the quantifiers as in the text, we have the desired result, which was proved by Addison and Kleene in $A$ note on function quantification, Proc. Amer. Math. Soc. 8 (1957), 1002-1006, using an argument involving the operation $A$.

\section{REFERENCES}

C. A. Church, The constructive second number class, Bull. Amer. Math. Soc. 44 (1938), 224-232.

CK. A. Church and S. C. Kleene, Formal definitions in the theory of ordinal numbers, Fund. Math. 28 (1937), 11-21.

$\mathrm{K}_{1}$. S. C. Kleene, Arithmetical predicates and function quantifiers, Trans. Amer. Math. Soc. 79 (1955), 193-213.

$\mathbf{K}_{2}$. - Introduction to metamathematics, Van Nostrand, New York, 1952.

KR. D. L. Kreider and H. Rogers, Jr., Constructive versions of ordinal number classes, Trans. Amer. Math. Soc. 100 (1961), 325-369.

P. H. Putnam, Uniqueness ordinals in higher constructive number classes, Essays on the foundations of mathematics, pp. 190-206, Magnes Press, Jerusalem, 1961.

MassachusetTs InSTItUTE of TeChNology 